\title{
QUASIRANDOM PERMUTATIONS ARE CHARACTERIZED BY 4-POINT DENSITIES
}

\author{
Daniel Král' and Oleg Pikhurko
}

\begin{abstract}
For permutations $\pi$ and $\tau$ of lengths $|\pi| \leq|\tau|$, let $t(\pi, \tau)$ be the probability that the restriction of $\tau$ to a random $|\pi|$-point set is (order) isomorphic to $\pi$. We show that every sequence $\left\{\tau_{j}\right\}$ of permutations such that $\left|\tau_{j}\right| \rightarrow \infty$ and $t\left(\pi, \tau_{j}\right) \rightarrow 1 / 4$ ! for every 4-point permutation $\pi$ is quasirandom (that is, $t\left(\pi, \tau_{j}\right) \rightarrow$ $1 /|\pi|$ ! for every $\pi)$. This answers a question posed by Graham.
\end{abstract}

\section{Introduction}

Roughly speaking, a combinatorial object is called quasirandom if it has properties that a random object has asymptotically almost surely. This notion has been defined for various structures such as tournaments [CG91a], set systems [CG91b], subsets of $\mathbb{Z} / n \mathbb{Z}$ [CG92], $k$-uniform hypergraphs [CG90, Gow06, Gow07, HT89, KRS02], groups [Gow08], etc.

In particular, quasirandomness has been extensively studied for graphs. Extending earlier results of Rödl [Rod86] and Thomason [Tho87], Chung et al. [CGW89] gave seven equivalent properties of graph sequences such that the sequence of random graphs $\left\{G_{n, 1 / 2}\right\}$ possesses them with probability one. These properties include densities of subgraphs, values of eigenvalues of the adjacency matrix or the typical size of the common neighborhood of two vertices. In particular, it follows from the results in [CGW89] that if the density of 4-vertex subgraphs in a large graph is asymptotically the same as in $G_{n, 1 / 2}$, then this is true for every fixed subgraph. Graham (see [Coo04, page 141]) asked whether a similar phenomenon also occurs in the case of permutations.

Let us state his question more precisely. Let $S_{k}$ consist of permutations on $[k]:=$ $\{1, \ldots, k\}$. We view each $\pi \in S_{k}$ as a bijection $\pi:[k] \rightarrow[k]$ and call $|\pi|:=k$ its

Daniel Král': Part of the work leading to this invention has received funding from the European Research Council under the European Union's Seventh Framework Programme (FP7/20072013)/ERC grant agreement no. 259385. Oleg Pikhurko: This author was supported by the European Research Council (grant agreement no. 306493) and the National Science Foundation of the USA (grant DMS-1100215).

Keywords and phrases: Permutations, Quasirandomness, Permutation limits, Subpermutation density 
length. For $\pi \in S_{k}$ and $\tau \in S_{m}$ with $k \leq m$, let $t(\pi, \tau)$ be the probability that a random $k$-point subset $X$ of $[m]$ induces a permutation isomorphic to $\pi$ (that is, $\tau\left(x_{i}\right) \leq \tau\left(x_{j}\right)$ iff $\pi(i) \leq \pi(j)$ where $X$ consists of $\left.x_{1}<\cdots<x_{k}\right)$. A sequence $\left\{\tau_{j}\right\}$ of permutations has Property $\mathbf{P}(k)$ if $\left|\tau_{j}\right| \rightarrow \infty$ and $t\left(\pi, \tau_{j}\right)=1 / k !+o(1)$ for every $\pi \in S_{k}$. It is easy to see that $\mathbf{P}(k+1)$ implies $\mathbf{P}(k)$. Graham asked whether there exists an integer $m$ such that $\mathbf{P}(m)$ implies $\mathbf{P}(k)$ for every $k$. Here we answer this question:

Theorem 1. Property $\mathbf{P}(4)$ implies Property $\mathbf{P}(k)$ for every $k$.

It is trivial to see that $\mathbf{P}(1) \nRightarrow \mathbf{P}(2)$ and an example that $\mathbf{P}(2) \nRightarrow \mathbf{P}(3)$ can be found in [Coo04]. An unpublished manuscript of Cooper and Petrarca [CP08] shows that $\mathbf{P}(3) \nRightarrow \mathbf{P}(4)$ and mentions that Chung could also show this (as early as 2001). Being unaware of [CP08], we found yet another example that $\mathbf{P}(3) \nRightarrow \mathbf{P}(4)$. Since it is quite different from the construction in [CP08], we present it in Section 4.

Since these notions deal with properties of sequences of permutations, we find it convenient to operate with an appropriately defined "limit object", analogous to that for graphs introduced by Lovász and Szegedy [LS06]. Here we use the analytic aspects of permutation limits that were studied by Hoppen et al. [HKMRS12,HKMS10] and we derive Theorem 1 from its analytic analog (Theorem 3).

Let the (normalized) discrepancy $d(\tau)$ of $\tau \in S_{n}$ be the maximum over intervals $A, B \subseteq[n]$ of

$$
\left|\frac{|A||B|}{n^{2}}-\frac{|\tau(A) \cap B|}{n}\right| .
$$

Cooper [Coo04] calls a permutation sequence $\left\{\tau_{j}\right\}$ quasirandom if $\left|\tau_{j}\right| \rightarrow \infty$ and $d\left(\tau_{j}\right) \rightarrow 0$. He also gives other equivalent properties [Coo04, Theorem 3.1] and he discusses various applications of "random-like" permutations. Using the results of [HKMRS12, HKMS10], it is not hard to relate quasirandomness and Properties $\mathbf{P}(k)$ :

Proposition 2. A sequence $\left\{\tau_{j}\right\}$ of permutations is quasirandom if and only if it satisfies Property $\mathbf{P}(k)$ for every $k$.

The proof of Proposition 2 can be found in Section 5. Thus our Theorem 1 implies that $\mathbf{P}(4)$ alone is equivalent to quasirandomness.

Finally, let us remark that McKay et al. [MMW02, pp. 121] also defined a notion of quasirandomness for permutations. Their definition, although related, is different from that of Cooper as it deals with sequences of sets of permutations.

\section{Limits of Permutations}

Here we define convergence of permutation sequences and show how a convergent sequence can be associated with an analytic limit object. We refer the reader to [HKMRS12, HKMS10] for more details. 
Let $\mathcal{Z}$ consist of probability measures $\mu$ on the Borel $\sigma$-algebra of $[0,1]^{2}$ that have uniform marginals, that is, $\mu(A \times[0,1])=\mu([0,1] \times A)=\lambda(A)$ for every Borel set $A \subseteq[0,1]$, where $\lambda$ is the Lebesgue measure on $[0,1]$.

Fix some $\mu \in \mathcal{Z}$. Let $V_{i}=\left(X_{i}, Y_{i}\right)$ for $i \in[k]$ be independent random variables with $V_{i} \sim \mu$ (that is, each $V_{i}$ has distribution $\mu$ ). We view an outcome $\left(X_{1}, Y_{1}, \ldots, X_{k}, Y_{k}\right)$ as an element of $[0,1]^{2 k}$. For permutations $\pi, \tau \in S_{k}$, let $A_{\pi, \tau} \subseteq[0,1]^{2 k}$ correspond to the event that

$$
X_{i}<X_{j} \text { iff } \pi(i)<\pi(j) \quad \& \quad Y_{i}<Y_{j} \text { iff } \tau(i)<\tau(j)
$$

(for example, the first statement above is equivalent to $\left.X_{\pi^{-1}(1)}<\cdots<X_{\pi^{-1}(k)}\right)$. Since each of the vectors $\left(X_{1}, \ldots, X_{k}\right)$ and $\left(Y_{1}, \ldots, Y_{k}\right)$ is uniformly distributed over $[0,1]^{k}$, the probability of the degenerate event

$$
D_{k}:=\left\{X_{i}=X_{j} \text { or } Y_{i}=Y_{j} \text { for some } i \neq j\right\} \subseteq[0,1]^{2 k}
$$

is zero. Note that the sets $A_{\pi, \tau}$ for $\pi, \tau \in S_{k}$ partition $[0,1]^{2 k} \backslash D_{k}$. If we reorder the indices in an outcome $\left(V_{1}, \ldots, V_{k}\right) \in[0,1]^{2 k} \backslash D_{k}$ so that $X_{1}<\cdots<X_{k}$, then the new relative order on $Y_{1}, \ldots, Y_{k} \in[0,1]$ defines a random permutation $\sigma(k, \mu) \in S_{k}$. In other words, if we land in $A_{\pi, \tau}$, then we set $\sigma(k, \mu)=\tau \pi^{-1}$. Let the density $t(\pi, \mu)$ of $\pi \in S_{k}$ be the probability that $\sigma(k, \mu)=\pi$. Equivalently,

$$
t(\pi, \mu)=\sum_{\rho \in S_{k}} \mu^{k}\left(A_{\rho, \pi \rho}\right)=k ! \mu^{k}\left(A_{\tau, \pi \tau}\right), \quad \text { any } \tau \in S_{k},
$$

where the last equality uses the fact that $\mu^{k}\left(A_{\rho, \pi \rho}\right)$ does not depend on $\rho \in S_{k}$ (because $V_{1}, \ldots, V_{k}$ are independent and identically distributed).

A sequence of permutations $\left\{\tau_{j}\right\}$ is convergent if $\left|\tau_{j}\right| \rightarrow \infty$ and $\left\{t\left(\pi, \tau_{j}\right)\right\}$ converges for every permutation $\pi$. This is the same definition of convergence as the one in [HKMRS12,HKMS10] except we additionally require that $\left|\tau_{j}\right| \rightarrow \infty$; cf. [HKMRS12, Claim 2.4].

It is easy to show that every sequence of permutations whose lengths tend to infinity has a convergent subsequence; see e.g. [HKMS10, Lemma 2.11]. Furthermore, for every convergent sequence $\left\{\tau_{j}\right\}$ there is $\mu \in \mathcal{Z}$ such that for every permutation $\pi$ we have

$$
\lim _{j \rightarrow \infty} t\left(\pi, \tau_{j}\right)=t(\pi, \mu)
$$

For the reader's convenience, we sketch the proof from [HKMRS12] that $\mu$ exists. For $\pi \in S_{k}$, let $\mu_{\pi} \in \mathcal{Z}$ be obtained by dividing the square $[0,1]^{2}$ into $k \times k$ equal squares and distributing the mass uniformly on the squares with indices $(i, \pi(i)), i=1, \ldots, k$. By Prokhorov's theorem, $\left\{\mu_{\tau_{j}}\right\}$ has a subsequence that weakly converges to some measure $\mu$. We have $\mu \in \mathcal{Z}$ as this set is closed in the weak topology. Finally, $\mu$ satisfies (3) because, for any fixed $\pi$, the function $t(\pi,-): \mathcal{Z} \rightarrow \mathbb{R}$ is continuous in the weak topology and $t\left(\pi, \tau_{j}\right)=t\left(\pi, \mu_{\tau_{j}}\right)+O\left(1 /\left|\tau_{j}\right|\right)$. 
We remark that Hoppen et al. [HKMRS12, HKMS10] proposed a slightly different limit object: the regular conditional distribution function of $Y$ with respect to $X$, where $(X, Y) \sim \mu$. Lemma 2.2 and Definition 2.3 in [HKMRS12] show how to switch back and forth between the two objects.

Now, we are ready to state the analytic version of Theorem 1 . Let us call $\mu \in \mathcal{Z}$ $k$-symmetric if $t(\pi, \mu)=1 / k$ ! for every $\pi \in S_{k}$.

Theorem 3. Every 4-symmetric $\mu \in \mathcal{Z}$ is the (uniform) Lebesgue measure on $[0,1]^{2}$. In particular, $\mu$ is $k$-symmetric for every $k$.

Let us show how Theorem 3 implies Theorem 1. Suppose on the contrary that some $\left\{\tau_{j}\right\}$ satisfies $\mathbf{P}(4)$ but not $\mathbf{P}(k)$. Fix $\pi \in S_{k}$ and a subsequence $\left\{\tau_{j}^{\prime}\right\}$ such that $\lim _{j \rightarrow \infty} t\left(\pi, \tau_{j}^{\prime}\right)$ exists and is not equal to $1 / k$ !. Consider now a convergent subsequence $\left\{\tau_{j}^{\prime \prime}\right\}$ of $\left\{\tau_{j}^{\prime}\right\}$ and let $\mu \in \mathcal{Z}$ be its limit. By (3), $\mu$ is 4-symmetric and, by Theorem $3, \mu$ is $m$-symmetric for every $m$. But then $\lim _{j \rightarrow \infty} t\left(\pi, \tau_{j}^{\prime \prime}\right)=t(\pi, \mu)=1 / k$ !, which is the desired contradiction.

\section{Proof of Theorem 3}

In this section, let $\mu \in \mathcal{Z}$ be arbitrary with $t(\pi, \mu)=1 / 4$ ! for every $\pi \in S_{4}$. Let $\lambda \in \mathcal{Z}$ denote the uniform measure on $[0,1]^{2}$. Our objective is to show that $\mu=\lambda$.

Let $V=(X, Y) \sim \mu$ and $v=(x, y) \sim \lambda$ be independent. For brevity, let us abbreviate $\int_{[0,1]^{2}}$ to $\int$. Define a function $F:[0,1]^{2} \rightarrow[0,1]$ by

$$
F(a, b):=\mu([0, a] \times[0, b])=\int_{V \leq(a, b)} \mathrm{d} V,
$$

where $V \leq(a, b)$ means that $X \leq a$ and $Y \leq b$. Since $\mu$ has uniform marginals, the function $F$ is continuous.

First, we show that the 4-symmetry of $\mu$ uniquely determines certain integrals.

LEMMA 4.

$$
\int F(X, Y)^{2} \mathrm{~d} V=\int F(X, Y) X Y \mathrm{~d} V=\int F(x, y)^{2} \mathrm{~d} v=\frac{1}{9} .
$$

Proof. Let $V_{i}=\left(X_{i}, Y_{i}\right) \sim \mu$, for $i=1,2, \ldots$, be independent random variables distributed according to $\mu$. By Fubini's theorem, we have

$$
\int F(X, Y)^{2} \mathrm{~d} V=\int\left(\int_{V_{2} \leq V_{1}} \mathrm{~d} V_{2}\right)\left(\int_{V_{3} \leq V_{1}} \mathrm{~d} V_{3}\right) \mathrm{d} V_{1}=\int_{A} \mathrm{~d}\left(V_{1}, V_{2}, V_{3}\right),
$$

where $A=\left\{\left(V_{1}, V_{2}, V_{3}\right): V_{2} \leq V_{1}\right.$ and $\left.V_{3} \leq V_{1}\right\} \subseteq[0,1]^{6}$. Note that

$$
A \backslash D_{3}=\bigcup_{\substack{\pi, \tau \in S_{3} \\ \pi(1)=\tau(1)=3}} A_{\pi, \tau},
$$


where $D_{3}$ is defined by (1) and the union is over $\pi, \tau \in S_{3}$ such that $\pi(1)=\tau(1)=3$. The 4-symmetry of $\mu$ and (2) imply that $\mu^{k}\left(A_{\pi, \tau}\right)=(1 / k !)^{2}$ for every $k \leq 4$ and $\pi, \tau \in S_{k}$. Since $\mu^{3}\left(D_{3}\right)=0$, we have $\mu^{3}(A)=4 \cdot(1 / 3 !)^{2}=1 / 9$, as required.

Likewise,

$$
\int F(X, Y) X Y \mathrm{~d} V=\int_{B} \mathrm{~d}\left(V_{1}, \ldots, V_{4}\right)
$$

where $B \subseteq[0,1]^{8}$ corresponds to the event that $V_{2} \leq V_{1}, X_{3} \leq X_{1}$ and $Y_{4} \leq Y_{1}$. One can derive (4) by replacing each factor by an integral (for example, $X$ is replaced by $\left.\int_{X_{3} \leq X} \mathrm{~d} V_{3}\right)$ and applying Fubini's theorem.

The integral in the right-hand side of (4) is equal to the $\mu^{4}$-measure of the union of $A_{\pi, \tau}$ over some (explicit) set of pairs $\pi, \tau \in S_{4}$. The measure of this set is uniquely determined by the 4 -symmetry of $\mu$. Thus the integral does not change if we replace $\mu$ by any other 4-symmetric measure. Considering the uniform measure $\lambda$, we obtain $\int x^{2} y^{2} \mathrm{~d} v=1 / 9$, as required.

Next, observe that $\left(X_{1}, Y_{2}\right)$ is uniformly distributed in $[0,1]^{2}$ because $V_{1}$ and $V_{2}$ are independent and have uniform marginals. Again, the value of

$$
\int F(x, y)^{2} \mathrm{~d} v=\int_{[0,1]^{4}} F\left(X_{1}, Y_{2}\right)^{2} \mathrm{~d}\left(V_{1}, V_{2}\right)=\int_{V_{3}, V_{4} \leq\left(X_{1}, Y_{2}\right)} \mathrm{d}\left(V_{1}, \ldots, V_{4}\right),
$$

does not depend on the choice of $\mu$ and can be easily computed by taking $\mu=\lambda$.

Since $X$ is uniformly distributed in $[0,1]$, we have $\int X^{2} \mathrm{~d} V=1 / 3$. Also,

$$
\int F(x, y) x y \mathrm{~d} v=\int_{v \geq V} x y \mathrm{~d}(v, V)=\frac{1}{4} \int\left(1-X^{2}-Y^{2}+X^{2} Y^{2}\right) \mathrm{d} V .
$$

We use the above identities and apply the Cauchy-Schwartz inequality twice to get the following series of inequalities:

$$
\begin{aligned}
\frac{1}{81} & =\left(\int F(X, Y) X Y \mathrm{~d} V\right)^{2} \leq\left(\int F(X, Y)^{2} \mathrm{~d} V\right) \cdot\left(\int X^{2} Y^{2} \mathrm{~d} V\right) \\
& =\frac{1}{9}\left(4 \cdot \int F(x, y) x y \mathrm{~d} v-\int\left(1-X^{2}-Y^{2}\right) \mathrm{d} V\right) \\
& =\frac{1}{9}\left(4 \cdot \int F(x, y) x y \mathrm{~d} v-\frac{1}{3}\right) \\
& \leq \frac{4}{9} \sqrt{\int F(x, y)^{2} \mathrm{~d} v} \cdot \sqrt{\int x^{2} y^{2} \mathrm{~d} v}-\frac{1}{27}=\frac{1}{81} .
\end{aligned}
$$

Thus we have equality throughout. However, the last inequality is equality if and only if $F(a, b)$ is equal to a fixed multiple of $a b$ almost everywhere with respect to 
the uniform measure $\lambda$. Since $F$ is continuous and $F(1,1)=1$, we conclude that $F(a, b)=a b$ for all $(a, b) \in[0,1]^{2}$. Thus the measures $\mu$ and $\lambda$ coincide on all rectangles $[0, a] \times[0, b]$ and on the ring of their finite Boolean combinations. Since this ring generates the Borel $\sigma$-algebra on $[0,1]^{2}$, we have that $\mu=\lambda$ by the uniqueness statement of the Carathéodory Theorem. This proves Theorem 3.

REMARK 5. Our proof gives other sufficient conditions for $\mu=\lambda$. For example, it suffices to require that each of the three integrals of Lemma 4 is $1 / 9$. The proof of the lemma shows that, if desired, these integrals can be expressed as linear combinations of densities $t(\pi, \mu)$ for $\pi \in S_{4}$. The single identity $\left(\int F(x, y) x y \mathrm{~d} v\right)^{2}=$ $\frac{1}{9} \int F(x, y)^{2} \mathrm{~d} v$ is also sufficient for proving that $\mu=\lambda$; however, if written as a polynomial in terms of permutation densities (by mimicking the proof of Lemma 4), it involves 5-point permutations. Our method can give other sufficient conditions in this manner; the choice of which one to use may depend on the available information about the sequence.

REMARK 6 . Also, the argument of Lemma 4 shows that, for every polynomial $P(x, y)$ and $\mu \in \mathcal{Z}$, the value of $\int P(x, y) \mathrm{d} \mu(x, y)$ can be expressed as a linear combination of permutation densities. This observation combined with the Stone-Weierstrass Theorem gives the uniqueness of a permutation limit: if $\mu, \mu^{\prime} \in \mathcal{Z}$ have the same permutation densities, then $\mu=\mu^{\prime}$ (cf. [HKMRS12, Theorem 1.7]).

\section{$4 \mathrm{P}(3)$ does not Imply $\mathrm{P}(4)$}

First, we construct a 3 -symmetric measure $\mu \in \mathcal{Z}$ which is not 4-symmetric. For $a \in[0,1]$, let $M(a)$ be the set of all the points $(x, y) \in[0,1]^{2}$ such that $x+y \in$ $\{1-a / 2,1+a / 2, a / 2,2-a / 2\}$ or $y-x \in\{-a / 2, a / 2,1-a / 2, a / 2-1\}$. See Figure 1 for illustrations of this definition. Define $\mu_{a} \in \mathcal{Z}$ for $a \in[0,1]$ to be the permutation limit such that the mass is uniformly distributed on $M(a)$. Because of the symmetries of $\mu_{a}$ (invariance under the horizontal and vertical reflections), we have that $t\left(\pi, \mu_{a}\right)=1 / 6$ for every $\pi \in S_{3}$ if and only if $t\left(\operatorname{Id}_{3}, \mu_{a}\right)=1 / 6$, where $\operatorname{Id}_{3}$ is the identity 3-point permutation.

Routine calculations show that $t\left(\operatorname{Id}_{3}, \mu_{0}\right)=1 / 4$ and $t\left(\operatorname{Id}_{3}, \mu_{1}\right)=1 / 8$. Since $t\left(\operatorname{Id}_{3}, \mu_{a}\right)$ is continuous in $a$, there exists $b \in[0,1]$ such that $t\left(\operatorname{Id}_{3}, \mu_{b}\right)=1 / 6$. Moreover, $\mu_{b}$ is not 4 -symmetric. This can be verified directly; it also follows from Theorem 3 since $\mu_{b}$ is not the uniform measure.
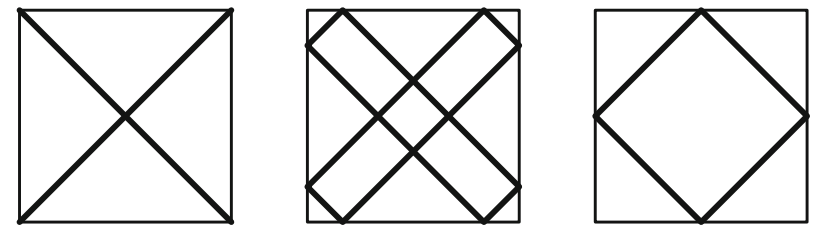

Figure 1: The sets $M(0), M(1 / 3)$ and $M(1)$ 
Take a sequence $\left\{\tau_{j}\right\}$ of permutations that converges to $\mu_{b}$. For example, the random sequence $\left\{\sigma\left(j, \mu_{b}\right)\right\}$ has this property with probability one, see [HKMS10, Corollary 4.3]. Any such sequence $\left\{\tau_{j}\right\}$ satisfies $\mathbf{P}(3)$ but not $\mathbf{P}(4)$.

REMARK 7. There are other ways how one can get an example of a 3 -symmetric non-uniform measure by transforming $M(0)$ into $M(1)$. For example, for $0<a<1$, let $\nu_{a} \in \mathcal{Z}$ assign measure $a$ to $M(0)$ and measure $1-a$ to $M(1)$ with the conditional distributions being equal to $\mu_{0}$ and $\mu_{1}$. Again by continuity, there is $a$ such that $\nu_{a}$ is 3-symmetric.

REMARK 8. Let us call a permutation $\pi \in S_{n} k$-inflatable if $n>1$ and $\mu_{\pi}$ is $k$-symmetric, where $\mu_{\pi} \in \mathcal{Z}$ is the measure associated with $\pi$ as is described after (3). Cooper and Petrarca [CP08] discovered many 3-inflatable permutations by computer search, thus giving examples that $\mathbf{P}(3) \nRightarrow \mathbf{P}(4)$. The results in [CP08] show that a shortest 3-inflatable permutation has length 9 and that $S_{9}$ has exactly four 3 -inflatable permutations: $(4,3,8,9,5,1,2,7,6),(4,7,2,9,5,1,8,3,6)$, and their vertical reflections. Clearly, our Theorem 3 implies that no 4 -inflatable permutation can exist. In particular, this proves (in a stronger form) Conjecture 3 in [CP08] that no 4-inflatable permutation with certain properties exists.

\section{Proof of Proposition 2}

Let $\left\{\tau_{j}\right\}$ be an arbitrary sequence of permutations with $\left|\tau_{j}\right| \rightarrow \infty$. Let $\mu_{j} \in \mathcal{Z}$ be the measure associated with $\tau_{j}$ as is described after (3). It is straightforward to verify that $d\left(\tau_{j}\right)=d\left(\mu_{j}\right)+o(1)$, where

$$
d(\mu):=\sup |\lambda(A \times B)-\mu(A \times B)|
$$

denotes the discrepancy of $\mu \in \mathcal{Z}$, with the supremum (in fact, it is maximum) being taken over intervals $A, B \subseteq[0,1]$. Also, it is not hard to show (cf. Remark 6 ) that $\left\{\tau_{j}\right\}$ converges to $\mu$ if and only if $\left\{\mu_{j}\right\}$ weakly converges to $\mu$.

First, suppose that $\left\{\tau_{j}\right\}$ satisfies $\mathbf{P}(k)$ for each $k$. This means that $\left\{\tau_{j}\right\}$ converges to the uniform limit $\lambda$. For $a, b \in[0,1]$, let $F_{j}(a, b):=\mu_{j}([0, a] \times[0, b])$ and $F(a, b):=a b$. Since $d(\lambda)=0$ and

$$
\mu_{j}\left(\left[a_{1}, a_{2}\right] \times\left[b_{1}, b_{2}\right]\right)=F_{j}\left(a_{2}, b_{2}\right)-F_{j}\left(a_{1}, b_{2}\right)-F_{j}\left(a_{2}, b_{1}\right)+F_{j}\left(a_{1}, b_{1}\right),
$$

we conclude that $d\left(\mu_{j}\right) \leq 4 \cdot\left\|F_{j}-F\right\|_{\infty}$. The weak convergence $\mu_{j} \rightarrow \lambda$ of measures in $\mathcal{Z}$ gives that $F_{j} \rightarrow F$ pointwise. Since $F$ and each function $F_{j}$, defined on the compact space $[0,1]^{2}$, are $\sqrt{2}$-Lipschitz, this implies that

$$
\left\|F_{j}-F\right\|_{\infty} \rightarrow 0
$$

(alternatively, (5) directly follows from [HKMRS12, Lemma 5.3]). Thus $d\left(\mu_{j}\right) \rightarrow 0$ and $\left\{\tau_{j}\right\}$ is quasirandom. 
Next suppose that $d\left(\tau_{j}\right) \rightarrow 0$. One way to establish Property $\mathbf{P}(k)$ is to use one of the equivalent definitions of quasirandomness from [Coo04, Theorem 3.1] (namely Property $[\mathrm{mS}])$. Alternatively, if $\mathbf{P}(k)$ fails, then (by passing to a subsequence) we can assume that $\left\{\tau_{j}\right\}$ converges to some $\mu \in \mathcal{Z}$ with $\mu \neq \lambda$. However, we have that $d(\mu)=0$, which implies $\mu=\lambda$, contradicting our assumption. This finishes the proof of Proposition 2.

\section{Concluding Remarks}

The theory of flag algebras developed by Razborov [Raz07] can be applied to permutation limits: a permutation $\pi: A \rightarrow A$ is viewed as two binary relations, each giving a linear order on $A$. For example, Lemma 4 can be stated and proved within the flag algebra framework. This view has been helpful for us when developing our proof.

A graph can be associated with a permutation $\pi \in S_{n}$ as follows: let $G(\pi)$ be the graph on $[n]$ with vertices $i<j$ adjacent if $\pi(i)<\pi(j)$. Fix $\mu \in \mathcal{Z}$ and sample a random permutation $\sigma(n, \mu)$. Define a function $W:[0,1]^{4} \rightarrow\{0,1\}$ by $W\left(x_{1}, y_{1}, x_{2}, y_{2}\right)=1$ if we have $\left(x_{1}, y_{1}\right)<\left(x_{2}, y_{2}\right)$ or $\left(x_{1}, y_{1}\right)>\left(x_{2}, y_{2}\right)$ componentwise and let $W\left(x_{1}, y_{1}, x_{2}, y_{2}\right)=0$ otherwise. In other words, $W$ is the indicator function of the event that $\sigma(2, \mu)$ is the identity 2-point permutation. Clearly, $G(\sigma(n, \mu))$ can be generated by sampling independently points $V_{1}, \ldots, V_{n} \in[0,1]^{2}$, each with distribution $\mu$, and connecting those $i, j \in[n]$ for which $W\left(V_{i}, V_{j}\right)=1$. The latter procedure corresponds to generating a random sample $\mathbb{G}(n, W)$, where $W:[0,1]^{2} \times[0,1]^{2} \rightarrow[0,1]$ is viewed as a graphon represented on Borel subsets of $[0,1]^{2}$ with measure $\mu$, see [LS06, Section 2.6] for details.

Lovász and Sós [LS08] and Lovász and Szegedy [LS11] presented various sufficient conditions for a graphon $W$ to be finitely forcible which, in the above notation, means that there is $m$ such that the distribution of $\mathbb{G}(m, W)$ uniquely determines that of $\mathbb{G}(k, W)$ for every $k$. As far as we can see, none of these conditions directly applies to the graphon associated with the uniform measure $\lambda \in \mathcal{Z}$. Since we answered Graham's question on quasirandom permutations by other means, we did not pursue this approach any further.

We also refer the reader to Hoppen et al. [HKMS11, Section 5.3] who discuss finite forcibility for permutation limits, being motivated by some questions in parameter testing.

\section{Acknowledgements}

The authors thank Carlos Hoppen, András Máthé, and the anonymous referee for helpful comments.

Open Access This article is distributed under the terms of the Creative Commons Attribution License which permits any use, distribution, and reproduction in any medium, provided the original author(s) and the source are credited. 


\section{References}

[CG90] F.R.K. Chung and R.L. Graham. Quasi-random hypergraphs. Random Structure and Algorithms, 1 (1990), 105-124.

[CG91a] F.R.K. Chung and R.L. Graham. Quasi-random tournaments. Journal of Graph Theory, 15 (1991), 173-198.

[CG91b] F.R.K. Chung and R.L. Graham. Quasi-random set systems. Journal of the American Mathematical Society, 4 (1991), 151-196.

[CG92] F.R.K. Chung and R.L. Graham. Quasi-random subsets of $\mathbb{Z}_{n}$. Journal of Combinatorial Theory, Series A, 61 (1992), 64-86.

[CGW89] F.R.K. Chung, R.L. Graham, and R.M. Wilson. Quasi-random graphs. Combinatorica, 9 (1989), 345-362.

[Coo04] J.N. Cooper. Quasirandom permutations. Journal of Combinatorial Theory, Series A, 106 (2004), 123-143.

[CP08] J.N. CoOper and A. Petrarca. Symmetric and asymptotically symmetric permutations, (2008) arXiv:0801.4181

[Gow06] W.T. Gowers. Quasirandomness, counting and regularity for 3-uniform hypergraphs. Combinatorics, Probability and Computing, 15 (2006), 143-184.

[Gow07] W.T. Gowers. Hypergraph regularity and the multidimensional Szemerédi theorem. Annals of Mathematics (2), 166 (2007), 897-946.

[Gow08] W.T. Gowers. Quasirandom groups. Combinatorics, Probability and Computing, 17 (2008), 363-387.

[HT89] J. Haviland, A.G. Thomason. Pseudo-random hypergraphs. Discrete Mathematics, 75 (1989), 255-278.

[HKMRS12] C. Hoppen, Y. Kohayakawa, C.G. Moreira, B. Ráth, and R.M. SamPAIO. Limits of permutation sequences. Journal of Combinatorial Theory, Series B, 103 (2012), 93-113.

[HKMS10] C. Hoppen, Y. Kohayakawa, C.G. Moreira, and R.M. Sampaio. Limits of permutation sequences through permutation regularity, (2010) arXiv:1106.1663

[HKMS11] C. Hoppen, Y. Kohayakawa, C.G. Moreira, and R.M. Sampaio. Testing permutation properties through subpermutations. Theoretical Computer Science, 412 (2011), 3555-3567.

[KRS02] Y. Kohayakawa, V. RöDL, and J. Skokan. Hypergraphs, quasi-randomness, and conditions for regularity. Journal of Combinatorial Theory, Series A, 97 (2002), 307-352.

[LS08] L. Lovász and V. Sós. Generalized quasirandom graphs. Journal of Combinatorial Theory, Series B, 98 (2008), 146-163.

[LS06] L. LovÁsz and B. SzEgedy. Limits of dense graph sequences. Journal of Combinatorial Theory, Series B, 96 (2006), 933-957.

[LS11] L. Lovász and B. Szegedy. Finitely forcible graphons. Journal of Combinatorial Theory, Series B, 101 (2011), 269-301.

[MMW02] B.D. MCKAY, J. Morse, and H.S. WILF. The distributions of the entries of Young tableaux. Journal of Combinatorial Theory, Series A, 97 (2002), $117-128$.

[Raz07] A. RAzBorov. Flag algebras. Journal of Symbolic Logic, 72 (2007), 1239-1282.

[Rod86] V. RöDL. On the universality of graphs with uniformly distributed edges. Discrete Mathematics, 59 (1986), 125-134. 
[Tho87] A. Thomason. Pseudo-random graphs. Annals of Discrete Mathematics, 33 (1987), 307-331.

DANIEL KRÁL', Mathematics Institute, DIMAP and Department of Computer Science, University of Warwick, Coventry CV4 7AL, UK

D.Kral@warwick.ac.uk Previous affiliation:

Faculty of Mathematics and Physics, Computer Science Institute, Charles University, Prague, Czech Republic

Oleg Pikhurko, Mathematics Institute and DIMAP, University of Warwick, Coventry, CV4 7AL, UK

0.Pikhurko@warwick.ac.uk

Received: May 14, 2012

Revised: January 11, 2013

Accepted: January 14, 2013 